\title{
Comparative efficacy and cost effectiveness of splenectomy and thrombopoietin prior to peginterferon and ribavirin therapy with compensatory cirrhosis associated with hepatitis $\mathbf{C}$ and thrombocytopenia
}

\author{
BING LI $^{1 *}$, YING JIE JI ${ }^{2 *}$, QING SHAO ${ }^{1}$, ZHENYU ZHU ${ }^{3}$, DONG JI ${ }^{1}$, FAN LI ${ }^{1}$ and GUOFENG CHEN ${ }^{1}$ \\ ${ }^{1}$ Liver Fibrosis Diagnosis and Treatment Center; ${ }^{2}$ Liver Disease Center for Military Staff; \\ ${ }^{3}$ Hepatobiliary Surgery Center, 302 Hospital of PLA, Beijing 100039, P.R. China
}

Received October 11, 2014; Accepted July 31, 2015

DOI: $10.3892 /$ etm.2015.2830

\begin{abstract}
The aim of the present study was to evaluate and compare the treatment efficacy and cost of two therapies, splenectomy and thrombopoietin, in order to optimize the treatment plans for patients with $\mathrm{HCV}$-associated cirrhosis. A prospective randomized controlled trial was conducted on 69 patients with a platelet count $<60,000 / \mathrm{mm}^{3}$ that were enrolled between 2009 and 2013, including 38 cases as the research group and 31 cases as the observed group. The study included two stages: A 4-week initial treatment and a 48-week antiviral treatment, during which a number of parameters were evaluated, including platelet count, liver stiffness measure, albumin, total bilirubin, alanine aminotranferase and treatment cost-effectiveness. Of the 38 patients, 21 underwent a splenectomy and their platelet counts increased to $60,000 / \mathrm{mm}^{3}$ after the 4-week initial treatment. The patients then started a 48-week P-R antiviral treatment, and 18 cases completed the treatment. In addition, 17/38 patients received thrombopoietin as a drug therapy. The platelet counts in 15 cases increased to $>60,000 / \mathrm{mm}^{3}$ and the patients received antiviral treatment, among which 9 cases completed the second treatment stage. The expense of the splenectomy group treatment was higher compared with that received by the thrombopoietin group. The results of the present study indicated that splenectomy was more effective at increasing platelet count. More splenectomy patients completed the full course of antiviral treatment and presented a sustained virologic response, compared with the thrombopoietin group. Therefore, splenectomy may be more expensive compared with thrombopoietin; however, the
\end{abstract}

Correspondence to: Professor Guofeng Chen, Liver Fibrosis Diagnosis and Treatment Center, 302 Hospital of PLA, 100 Xiansihuan Zhonglu, Beijing 100039, P.R. China

E-mail: gfchen302@163.com

${ }^{*}$ Contributed equally

Key words: thrombocytopenia, splenectomy, thrombopoietin, peginterferon, ribavirin improved efficacy suggests that on balance it is the preferable treatment option.

\section{Introduction}

Thrombocytopenia is a common complication of chronic liver disease and is considered to be an indicator of an advanced disease stage (1-3). Thrombocytopenia in patients with chronic hepatitis, indicated by a platelet count of $<60,000 / \mathrm{mm}^{3}$, has been associated primarily with two factors. One of these factors is hypersplenism resulting from splenomegaly in portal hypertension (4). Hypersplenism appears to be the most common cause of thrombocytopenia associated with liver cirrhosis and portal hypertension (5). The second mechanism is associated with the reduced production of thrombopoietin, a hormone produced by hepatocytes, which regulate the development of the megakaryocyte (5). In cirrhosis, due to the reduction in the mass of functioning hepatocytes, there may be a reduction of thrombopoiesis in the bone marrow, causing thrombocytopenia in the peripheral blood $(6,7)$.

In numerous situations, patients with thrombocytopenia that are infected with hepatitis $\mathrm{C}$ virus ( $\mathrm{HCV}$ ) cannot be treated with peginterferon and ribavirin (P-R) due to a low platelet count, which may jeopardize the treatment. $\mathrm{P}-\mathrm{R}$ is a routine treatment in $\mathrm{HCV}$ patients with thrombocytopenia and the only antiviral treatment available to these patients in China, as protease inhibitors are not approved (8). This is because oral anti-HCV drugs, such as protease inhibitor, are not typically covered by insurance schemes in China and are often prohibitively expensive for Chinese individuals. In the present study, splenectomy and thrombopoietin were used as two potential treatments to increase platelet count and assist patients in completing a full course of antiviral treatment. Furthermore, the negative effects and cost of the two methods were compared, with the aim of identifying the optimal therapy for patients in clinical treatment.

\section{Materials and methods}

Patients. Between June 2009 and May 2013, a total of 134 inpatients with HCV-associated compensated liver cirrhosis that 
were treated at the 302 Hospital of PLA (Beijing, China) were enrolled in the present study. The enrolled patients had indications of conditions requiring antiviral treatment, but had received no previous antiviral therapy. Among the initial 134 patients, 69 patients had a platelet count of $<60,000 / \mathrm{mm}^{3}$, of which 38 patients (Child-Pugh score A, 32; Child-Pugh score B, 6) were allocated to the research group and the other 31 patients to the observed group (9). All the patients supplied written informed consent. Eligible patients that had chronic HCV infection (defined as the presence of anti-HCV antibodies and detectable serum HCV RNA levels, as determined using a clinically-available assay selected by the investigator), compensated liver disease and thrombocytopenia (defined as a platelet count of $<60,000 / \mathrm{mm}^{3}$ ) were treated with antiviral treatment for 48 weeks. Cirrhosis of patients was met according to the hepatitis $\mathrm{C}$ cirrhosis diagnosis standard (10). Patients were excluded if they exhibited operative contraindication, absolute contraindications of interferon therapy or thrombocytopenia as a result of hematological system disease.

Written informed consent was obtained from all patients and the patient data was anonymized and de-identified prior to analysis. The study was approved by the Ethics Committee of the 302 Hospital of PLA (Beijing, China) and was conducted in accordance with the ethical standards formulated in the Declaration of Helsinki.

Study design. The present study was conducted as a prospective randomized controlled trial and was divided into two stages: Initial treatment phase and antiviral treatment phase, as described in a previous study (11). A total of 38 patients that met the eligibility criteria were randomly assigned to the splenectomy $(\mathrm{n}=21)$ and thrombopoietin (3SBio, Inc., Shenyang, China) ( $\mathrm{n}=17)$ groups. A further 31 patients were allocated to the observed group and treated with glycyrrhizin injection (100 ml per day; North China Pharmaceutical Co., Ltd., Shanghai, China), wuzhi capsule (3 capsules, twice per day; HYGIEN, Sichuan, China) and thymopentin injection (10 mg per day; Beijing SL Pharmaceutical Co., Ltd., Beijing, China).

Initial treatment phase. Patients received splenectomy or were administered thrombopoietin $(15,000$ units each time; subcutaneous injection), once per week or as determined by physicians according to patient platelet levels (Fig. 1). Parameters assessed to determine safety were prothrombin time, alanine aminotransferase, aspartate amino transferase, blood albumin, neutrophil count, creatinine clearance rate and hemoglobin, using an XE2100 hematology analyzer (Sysmex Shanghai Ltd., Shanghai, China) and an AU5800 biochemical analyzer (Beckman Coulter, Inc., Tokyo, Japan). Tolerability indices included fatigue, insomnia and depression. Patients that completed the initial treatment phase were subsequently administered antiviral treatment with peginterferon (Pegasys; Roche Diagnostics, Basel, Switzerland) and ribavirin (Biejing SL Pharmaceutical Co., Ltd., Beijing, China) if they attained a predefined platelet count of $\geq 60,000 / \mathrm{mm}^{3}$. The control group included 31 patients ( 23 men and 8 women) with an average age of 55 years (age range, 39-70 years). Patients in the control group did not receive treatments such as thrombopoietin and P-R.

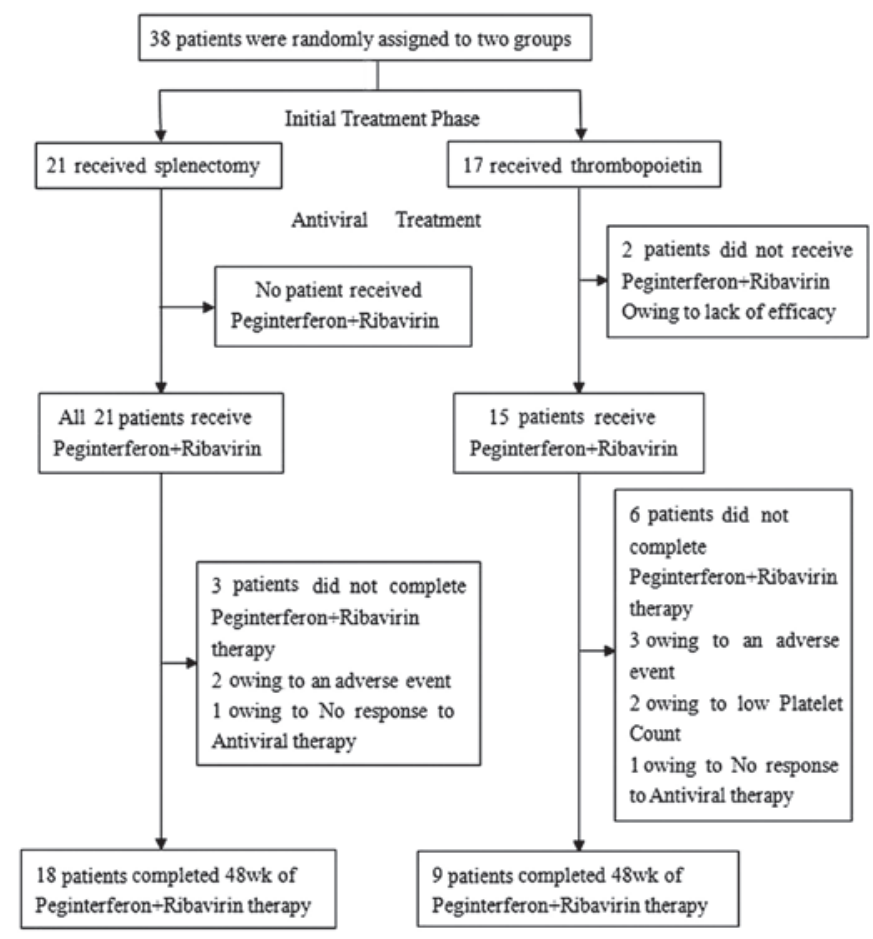

Figure 1. Randomization and follow-up of the study patients.

Antiviral treatment phase. Peginterferon (180 $\mu \mathrm{g}$ peginterferon alfa-2a per week) and ribavirin $(15 \mathrm{mg} / \mathrm{kg}$ body weight per day) were administered for 48 weeks concomitantly with hematological, biochemical and other safety assessments. If the treatment was insufficiently effective or caused severe side effects, antiviral treatment was immediately discontinued. Treatment follow-up was scheduled for 24 weeks after the final P-R treatment. Throughout the antiviral treatment phase, in accordance with the product labels for these approved therapies, the dose of P-R was reduced by $50 \%$ if the platelet count had decreased to $25,000-50,000 / \mathrm{mm}^{3}$ and was discontinued completely if the platelet count was $<25,000 / \mathrm{mm}^{3}$. Data were obtained in the form of questionnaire, including work income during hospitalization and lost income of accompanying family members, food and accommodation costs.

Statistical analysis. All statistical analyses were conducted using SPSS software, version 17.0 (SPSS, Inc., Chicago, IL, USA). The primary efficacy end-point was defined as an increase in the platelet count from the original value to $\geq 60,000 / \mathrm{mm}^{3}$ following the 4 -week initial treatment phase. Secondary end-points included those associated with safety, tolerability and the ability to continue P-R therapy during the antiviral treatment phase. The analyses included all patients that were randomly assigned to the splenectomy and thrombopoietin groups and received at least one treatment course of P-R. However, data for $2 / 69$ patients (3\%) that were enrolled into the study were excluded from the analysis of the primary end-point, since their platelet count remained at $\leq 60,000 / \mathrm{mm}^{3}$ after treatment.

The primary end-point was analyzed with the use of multiple logistic-regression analysis. Research group patients were compared with the observed group patients using a closed testing procedure. The global null hypothesis of no significant 
Table I. Baseline characteristics of the patients.

\begin{tabular}{|c|c|c|}
\hline Characteristic & Splenectomy $(\mathrm{n}=21)$ & Thrombopoietin $(\mathrm{n}=17)$ \\
\hline \multicolumn{3}{|l|}{ Age, years } \\
\hline Median & 52 & 57 \\
\hline Range & $40-66$ & $42-63$ \\
\hline \multicolumn{3}{|l|}{ Gender, n (\%) } \\
\hline Male & $17(81)$ & $14(82)$ \\
\hline Female & $4(19)$ & $3(18)$ \\
\hline \multicolumn{3}{|l|}{ Platelet count, $/ \mathrm{mm}^{3}$} \\
\hline Median & 39,000 & 39,000 \\
\hline Range & $21,000-59,000$ & $19,000-57,000$ \\
\hline \multicolumn{3}{|l|}{ Liver stiffness, $\mathrm{kPa}$} \\
\hline Median & 19.9 & 19.7 \\
\hline Range & $12.6-31.2$ & $13.4-29.7$ \\
\hline HCV RNA load, IU/ml & $5.1 \times 10^{5} \pm 2.3 \times 10^{3}$ & $4.9 \times 10^{5} \pm 2.4 \times 10^{3}$ \\
\hline \multicolumn{3}{|l|}{ HCV genotype, n (\%) } \\
\hline $1 b$ & $16(76)$ & $12(71)$ \\
\hline $2 \mathrm{a}$ & $5(24)$ & $5(29)$ \\
\hline Alanine aminotransferase, IU/1 & $48.3 \pm 19.6$ & $47.9 \pm 15.3$ \\
\hline Aspartate aminotransferase, IU/1 & $50.7 \pm 22.6$ & $52.1 \pm 16.3$ \\
\hline Total bilirubin, $\mu \mathrm{mol} / 1$ & $21.1 \pm 12.4$ & $19.8 \pm 13.5$ \\
\hline \multicolumn{3}{|l|}{ Child-Pugh score, n (\%) } \\
\hline A & $17(81)$ & $15(88)$ \\
\hline $\mathrm{B}$ & $4(19)$ & $2(12)$ \\
\hline
\end{tabular}

Plus-minus values are presented the mean \pm standard deviation. No statistically significant differences were observed in characteristics between two study groups $(\mathrm{P}>0.05)$. $\mathrm{HCV}$, hepatitis $\mathrm{C}$ virus.

difference was tested between the two groups. The criterion for stopping the study early was a two-sided P-value $\leq 0.0001$, based on the O'Brien-Fleming adjustment for a group sequential design, from an interim analysis of the efficacy data (12). $\mathrm{P}<0.05$ was considered to indicate a statistically significant difference.

\section{Results}

Patient characteristics. A total of 69 patients were included in the study between 2009 and 2013, among which 38 patients were randomly assigned to the research group. Among these 38 patients, 21 underwent a splenectomy and 17 received thrombopoietin (Fig. 1). A further 31 patients were allocated to the observed group and treated with glycyrrhizin, legalon, Chinese herbs and thymic peptide. The demographic and baseline clinical characteristics of the research groups were well-balanced, with no statistically significant differences between the groups (Table I). The median patient ages were 52 years (range, 40-66 years) and 57 years (range, $42-63$ years) for the splenectomy and thrombopoietin groups, respectively, and $>80 \%$ of the patients were men.

The median original platelet count was $39,000 / \mathrm{mm}^{3}$ (range, $19,000-59,000 / \mathrm{mm}^{3}$ ) and all platelet counts were $<60,000 / \mathrm{mm}^{3}$ (Table I). However, in $2 / 38$ patients, the platelet count was not successfully increased to $\geq 60,000 / \mathrm{mm}^{3}$ following treatment, which was considered a violation of the study inclusion criteria. Therefore, the data for these 2 patients were excluded from the analysis of the antiviral treatment phase; however, they were included in the safety analysis as the patients received the various study therapies. The observed group (control group), that received no treatments such as thrombopoietin and P-R, had a platelet count of $36,000 / \mathrm{mm}^{3}$ (range, $23,000-56,000 / \mathrm{mm}^{3}$ ). During the 72 -week observed period, the results showed 5 cases of mortality, 4 cases of upper digestive tract bleeding, 1 cases of hepatic encephalopathy and 3 cases of hepatocellular carcinoma.

\section{Efficiency}

Initial treatment phase. The receipt of splenectomy or thrombopoietin increased platelet counts to $\geq 65,000 / \mathrm{mm}^{3}$ at week 4 in all patients, with the exception of two patients that received thrombopoietin $(\mathrm{P}<0.005$ for overall treatment effect; Table II). A total of 18 patients $(86 \%)$ that underwent a splenectomy had platelet counts of $\geq 100,000 / \mathrm{mm}^{3}$ at all time points during the initial treatment phase, thus follow-up to the antiviral treatment required cautious observation.

Antiviral treatment phase. Overall, 36/38 patients proceeded to the antiviral treatment phase, including $21 / 21$ patients $(100 \%)$ that received a splenectomy and 15/17 (88\%) 
Table II. Median platelet counts at the initial treatment phase and the end of the antiviral treatment phase.

A, End of initial treatment phase

\begin{tabular}{lcc}
\hline Variable & Splenectomy $(\mathrm{n}=21)$ & Thrombopoietin $(\mathrm{n}=17)$ \\
\hline Platelet count ${ }^{\mathrm{a}}, \mathrm{mm}^{3}$ & & 68,000 \\
Median & 204,000 & $29,000-89,000$ \\
Range & $74,000-365,000$ & 25,000 \\
Difference from baseline ${ }^{\mathrm{a}}$ & & $6,000-42,000$ \\
Median, $/ \mathrm{mm}^{3}$ & 155,000 & $15 / 17(88)$ \\
Range, $/ \mathrm{mm}^{3}$ & $33,000-335,000$ & $0 / 17(0)^{\mathrm{a}}$ \\
$\geq 60,000 / \mathrm{mm}^{3}, \mathrm{n}(\%)$ & $21 / 21(100)$ & \\
Responders $/ \mathrm{total}^{3}$ patients, $\mathrm{n}(\%)$ & $18 / 21(86)$ & \\
$\geq 100,000 / \mathrm{mm}^{3}$ & & \\
\hline
\end{tabular}

$\mathrm{B}$, End of antiviral treatment phase

Variable

Splenectomy $(n=21)$

Thrombopoietin $(\mathrm{n}=17)$

Platelet count

No. patients, n (\%)

$18(86)$

$9(53)$

Median, $/ \mathrm{mm}^{3}$

96,000

56,000

Range, $/ \mathrm{mm}^{3}$

$54,000-251,000$

$44,000-92,000$

Difference from baseline, $/ \mathrm{mm}^{3}$

Median

68,000

16,000

Range

$-3,000-198,000$

$-7,000-60,000$

${ }^{\text {a }} \mathrm{P}<0.005$ for patients whose platelet count increased to $60,000 / \mathrm{mm}^{3}$ in the splenectomy and thrombopoietin groups at the end of the initial treatment phase.

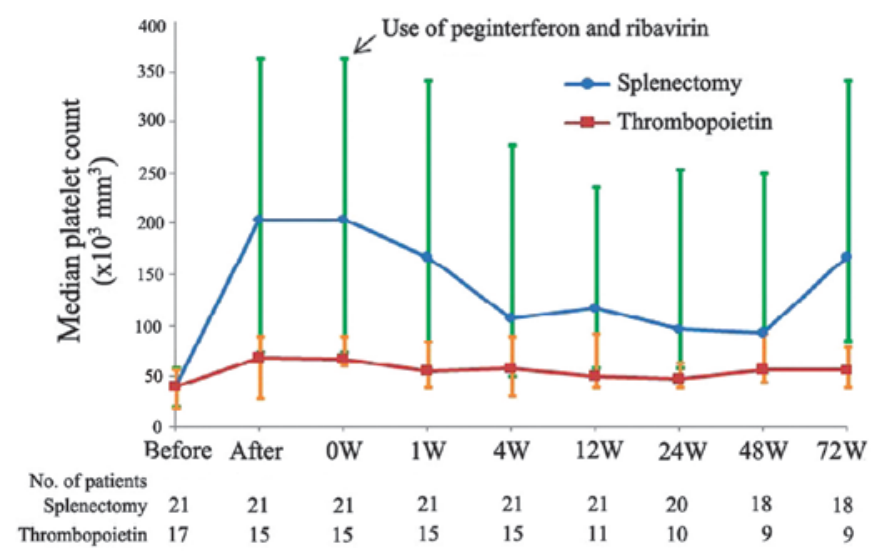

Figure 2. Median platelet counts and the number of patients that completed the 72 -week observed period. W, week.

that received thrombopoietin (Fig. 1). The platelet counts of these 36 patients all attained the prespecified threshold for entry into the antiviral treatment phase $\left(\geq 60,000 / \mathrm{mm}^{3}\right)$ and therefore P-R-based therapy was initiated (Fig. 1).

On an intention-to-treat basis, the first 12 weeks of antiviral therapy were completed by $21 / 21$ patients that underwent a splenectomy $(100 \%)$ and $11 / 15$ patients that received thrombopoietin (73\%; Fig. 2). Platelet counts in the splenectomy and thrombopoietin groups decreased during the antiviral treatment phase, but remained consistently above baseline values, with a nadir of $>60,000 / \mathrm{mm}^{3}$ (Fig. 2). In total, 18/21 patients $(86 \%)$ from the splenectomy group and $9 / 11$ patients $(82 \%)$ from the thrombopoietin treatment group completed the 48-week antiviral treatment phase. At all time points during the antiviral treatment phase, platelet counts in the splenectomy group were high and remained elevated compared with the level at which a reduction in the P-R dose is recommended $\left(<50,000 / \mathrm{mm}^{3}\right)$. During the 72 -week observed period, the percentage of completed antiviral treatment was $85.7 \%(\mathrm{P}=0.0636)$, the rapid virological response was $28.6 \%(\mathrm{P}=0.3879)$, the early virological response was $76.2 \%(\mathrm{P}=0.0272)$ and the sustained virologic response (SVR) was $61.9 \%(\mathrm{P}=0.0416)$ in splenectomy group, whereas the values in the thrombopoietin group were $52.9,11.8,35.3$ and $23.5 \%$, respectively. In addition, the differences in liver stiffness measure, albumin, total bilirubin and alanine aminotransferase are presented in Fig. 3.

Safety and tolerability. During the 4-week initial treatment phase, headache was reported in 5 and $24 \%$ of patients that underwent splenectomy or received thrombopoietin, respectively (Table III). Other common adverse events in the two research groups were upper abdominal pain, pyrexia, rash and nausea. During the subsequent antiviral treatment phase, the incidence of adverse events was similar between the two 
Table III. Common adverse events during the initial treatment phase and the antiviral phase [n (\%)].

\begin{tabular}{|c|c|c|}
\hline Events & $\begin{array}{l}\text { Splenectomy } \\
\quad(n=21)\end{array}$ & $\begin{array}{l}\text { Thrombopoietin } \\
\qquad(\mathrm{n}=17)\end{array}$ \\
\hline \multicolumn{3}{|l|}{ Initial treatment phase } \\
\hline Any & $16(76)$ & $12(71)$ \\
\hline Headache & $1(5)$ & $4(24)$ \\
\hline Upper abdominal pain & $5(24)$ & $0(0)$ \\
\hline Pyrexia & $6(29)$ & $4(24)$ \\
\hline Nausea & $4(19)$ & $1(6)$ \\
\hline Rash & $0(0)$ & $3(18)$ \\
\hline \multicolumn{3}{|l|}{ Antiviral treatment phase } \\
\hline Any & $20(95)$ & $14(93)$ \\
\hline Influenza-like illness & $13(62)$ & $11(73)$ \\
\hline Fatigue & $8(38)$ & $6(40)$ \\
\hline Headache & $3(14)$ & $9(60)$ \\
\hline Arthralgia & $3(14)$ & $4(27)$ \\
\hline Depression & $2(10)$ & $2(13)$ \\
\hline Myalgia & $3(14)$ & $2(13)$ \\
\hline Nausea & $3(14)$ & $4(27)$ \\
\hline Anemia & $12(57)$ & $10(67)$ \\
\hline Pyrexia & $8(38)$ & $10(67)$ \\
\hline Diarrhea & $4(19)$ & $3(20)$ \\
\hline Irritability & $5(24)$ & $4(27)$ \\
\hline Pruritus & $3(14)$ & $5(33)$ \\
\hline Rash & $2(10)$ & $7(47)$ \\
\hline
\end{tabular}

The general and each project incidence of adverse reaction had no significant difference in two stages of treatment.

Table IV. Reasons for not completing therapy [n (\%)].

\begin{tabular}{lcc}
\hline Variable & $\begin{array}{c}\text { Splenectomy } \\
(\mathrm{n}=21)\end{array}$ & $\begin{array}{c}\text { Thrombopoietin } \\
(\mathrm{n}=17)\end{array}$ \\
\hline Patients & $3(14)$ & $8(47)$ \\
Wuxiao & $1(5)$ & $1(6)$ \\
Platelet count & $0(0)$ & $4(24)$ \\
Fatigue & $1(5)$ & $2(12)$ \\
Depression & $1(5)$ & $0(0)$ \\
Rash & $0(0)$ & $1(6)$ \\
\hline
\end{tabular}

research groups. The adverse events most commonly reported during this phase were influenza-like illness, anemia, fatigue, pyrexia and irritability, all of which are known side-effects of P-R-based therapy $(13,14)$.

During the entire study, 24 patients reported adverse events and 5 patients reported serious adverse events. These serious events included ascites and depression in the splenectomy group, as well as severe asthenia, rash and upper gastrointestinal bleeding in the thrombopoietin group, resulting in the patients discontinuing therapy. Among these patients, 3 patients discontinued the P-R treatment, 1 due to ineffective
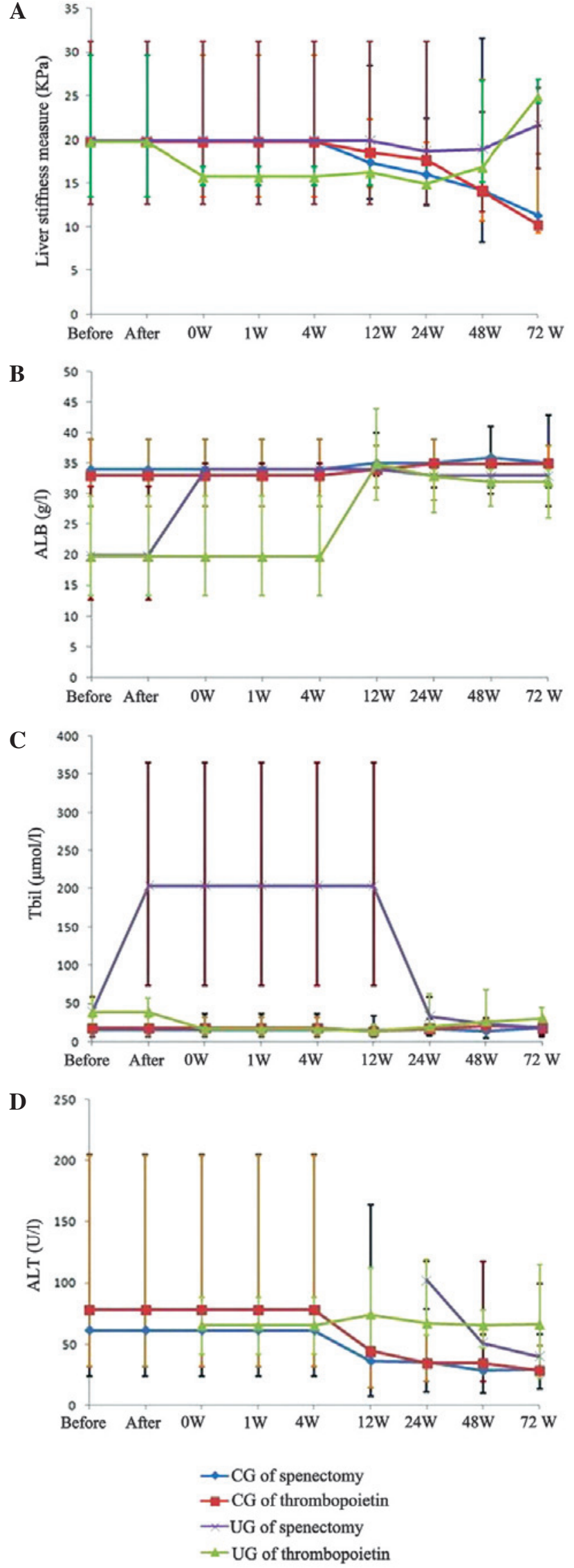

Figure 3. Differences in liver stiffness measure, ALB, Tbil and ALT during the 72-week observed period. (A) Result of liver stiffness measure during initial treatment phase and 72-week observed period. Differences in (B) ALB, (C) Tbil and (D) ALT during initial treatment phase and 72-week observed period. ALB, albumin; Tbil, total bilirubin; ALT, alanine aminotransferase; W, week; CG, completed group; UG, uncompleted group. 
Table V. Cost-effectiveness analysis at the percent of initial treatment and the end of the antiviral treatment.

\begin{tabular}{lcc}
\hline Variable & Splenectomy $(\mathrm{n}=21)$ & Thrombopoietin $(\mathrm{n}=17)$ \\
\hline Initial treatment phase & & $16,000(9,800-31,000)$ \\
Cost, RMB (Yuan) & $59,000(49,000-78,000)$ & 88 \\
Effectiveness, $\%$ & 100 & 3,583 \\
Incremental cost-effectiveness, $\Delta \mathrm{C} / \Delta \mathrm{E}$ & & $126,000(102,000-157,000)$ \\
Antiviral treatment phase & $85,000(78,000-93,000)$ & 53 \\
Cost, RMB (Yuan) & 86 & 879 \\
Effectiveness, $\%$ & & \\
Incremental cost-effectiveness, $\Delta \mathrm{C} / \Delta \mathrm{E}$ & & \\
\hline
\end{tabular}

$\mathrm{RMB}$, Renminbi; $\Delta \mathrm{C} / \Delta \mathrm{E}$, increase of cost/increase of effect ratio.

treatment and 2 due to serious adverse effects, including fatigue, skin itching or severe skin rash. Furthermore, 6 patients in the thrombopoietin group stopped treatment, 1 due to inefficacy, 2 due to severe fatigue of and 3 due to low platelet counts. Furthermore, 1 patient in the splenectomy group developed liver cancer following SVR and ceased therapy 7 months later. In addition, the Child-Pugh score of 4 patients (splenectomy, 3; thrombopoietin, 1) changed from A to B after the 48 -week therapy, and from B to A in another 3 patients in the thrombopoietin group prior to antiviral treatment. The causes for patients discontinuing the treatment are presented in Table IV.

Cost. As the enzyme inhibitor drugs are not typically covered by insurance schemes in China and are often prohibitively expensive, splenectomy, thrombopoietin and internal medicine are the three primary methods used for the treatment of compensatory cirrhosis associated with $\mathrm{HCV}$ and complicated with thrombocytopenia in China. Cost and efficacy are crucial factors for patients when selecting a treatment option. During the initial treatment phase the median cost of splenectomy was 59,000 RMB (range, 49,000-78,000 RMB) and the rate of effectiveness was $100 \%$. During the antiviral treatment phase, the median cost was 85,000 RMB (range, 78,000-93,000 RMB) and the effectiveness was $86 \%$ (Table V). A total of 31 patients in the observed group were uncertain factors such as ascites and bleeding caused by the treatment with glycyrrhizin, legalon, Chinese herbs and thymic peptide. The median cost of treatment was 95,000 RMB (range, 37,000-190,000 RMB). However, follow-up analysis of the observed group after 72 -week revealed that 6 patients exhibited decompensation, 3 patients had large ascites, 2 patients presented upper gastrointestinal bleeding, 1 case had hepatic encephalopathy and 3 cases had developed hepatocellular carcinoma. In addition, the Child-Pugh Score of 1 patient changed from A to B, and those of another 6 patients turned from B to A. The cost included all expenses incurred during the hospitalization period, including the living expenses of the patient and accompanying persons.

\section{Discussion}

Thrombocytopenic patients with chronic HCV infection are poor candidates for antiviral treatment with P-R (15); however, there is little information concerning the eligibility for treatment of patients with platelet counts of $<60,000 / \mathrm{mm}^{3}$. In the present study, $36 / 38$ patients exhibited a response to splenectomy or thrombopoietin, with an increase in platelet count to $\geq 60,000 / \mathrm{mm}^{3}$ during the initial treatment phase. These patients completed 48 weeks of antiviral treatment with P-R and 24-week follow-up, during which time their platelet count was monitored.

Splenectomy is a safe, efficacious and cost-effective intervention for patients with compensatory cirrhosis associated with $\mathrm{HCV}$. The International Consensus panel and the revised American Society of Hematology guidelines suggest deferral of splenectomy for as long as possible or to at least until 12 months of disease duration in patients with severe disease $(16,17)$. This caution is based on several concerns, including the high spontaneous remission rate, long-term risk for overwhelming post-splenectomy infection, increasing available medical alternatives (such as thrombopoietin agonists), and more recent concerns for vascular or hypercoagulable delayed complications (16-21).

Thrombopoietin, which is also known as megakaryocyte growth and development factor, is a protein that is encoded by the thrombopoietin gene in humans (22). Patients with cirrhosis usually exhibit thrombocytopenia in discrete levels. The mechanism of thrombocytopenia is hypothesized to involve the splenic sequestration and destruction of platelets, impaired bone marrow generation and diminished hepatic thrombopoietin synthesis (23).

The most commonly observed side effects of splenectomy and thrombopoietin during the initial treatment phase were headache, upper abdominal pain, pyrexia, nausea and rash; these effects were of insufficient severity to require discontinuation of the antiviral treatment (24). In the present study, no evidence of a dose-response association with respect to the occurrence of adverse events was observed in the antiviral treatment phase, during which the reported side effects were consistent with those associated with P-R-based therapy (11).

During the initial treatment phase, significant increases in platelet count were observed in each research group as compared with the original count. The primary end-point (platelet count of $\geq 60,000 / \mathrm{mm}^{3}$ at week 4) was attained in 100 and $88 \%$ of patients in the splenectomy and thrombopoietin groups, respectively. During the subsequent antiviral 
phase, platelet counts decreased, which may be due to the antiplatelet effect of P-R; however, the platelet counts remained consistently above the baseline levels.

The results of the present study indicate that splenectomy is more effective treatment for thrombocytopenia compared with thrombopoietin for facilitating the subsequent completion of antiviral therapy in patients with compensatory cirrhosis associated with HCV. In the splenectomy group, more patients were able to receive a full course of antiviral therapy and achieved an SVR. Follow-up demonstrated that the patients were in good condition. Side effects were observed in the two groups due to the P-R treatment or other factors, depending on each individual's physical condition. However, the expenses incurred by patients in the splenectomy group were higher than those in the thrombopoietin group due to the surgery fees, artificial nursing fees and other associated costs. In general, the therapeutic efficiency of the treatment is the highest priority for patients, and cost is the second priority, as long as it is within acceptable levels. Currently, splenectomy is considered a good thrombocytopenia treatment for assisting the completion of antiviral therapy in patients with compensatory cirrhosis associated with HCV. However, for patients that are able to afford the expenses associated with, the application of ribavirin therapy may result in higher efficacy with reduced risk.

\section{Acknowledgements}

This study was supported by the Chinese Medical Association Foundation (project no. 13071110496: Polyethylene Glycol Interferon- $\alpha-2 b$ plus Ribavirin for Treatment of Hepatitis $C$ in Patients with Cirrhosis). The authors would like to thank all participants who volunteered for this study, and particularly thank Professor George Ka Kit Lau (Li Ka Shing Faculty of Medicine, University of Hong Kong, Hong Kong, China).

\section{References}

1. Bashour FN, Teran JC and Mullen KD: Prevalence of peripheral blood cytopenias (hypersplenism) in patients with nonalcoholic chronic liver disease. Am J Gastroenterol 95: 2936-2939, 2000.

2. Peck-Radosavljevic M: Thrombocytopenia in liver disease. Can J Gastroenterol 14 (Suppl D): 60D-66D, 2000.

3. Giannini EG: Review article: Thrombocytopenia in chronic liver disease and pharmacologic treatment options. Aliment Pharmacol Ther 23: 1055-1065, 2006.

4. Moreno A, Bárcena R, Blázquez J, Quereda C, Gil-Grande L, Sánchez J, Moreno L, Perez-Elías MJ, Antela A, Moreno J, et al: Partial splenic embolization for the treatment of hypersplenism in cirrhotic HIV/HCV patients prior to pegylated interferon and ribavirin. Antivir Ther 9: 1027-1030, 2004.

5. de Oliveira AC: Treatment options in the management of thrombocytopenia in patients infected with HCV. Braz J Infect Dis 11 (Suppl 1): 71-72, 2007.

6. Fried MW, Shiffman ML, Reddy KL, Smith C, Marinos G, Gonçales FL Jr, Häussinger D, Diago M, Carosi G, Dhumeaux D, et al: Peginterferon alfa-2a plus ribavirin for chronic hepatitis C virus infection. N Engl J Med 347: 975-982, 2002.
7. Manns MP, McHutchison JG, Gordon SC, Rustgi VK, Shiffman M, Reindollar R, Goodman ZD, Koury K, Ling M and Albrecht JK: Peginterferon alfa-2b plus ribavirin compared with interferon alfa-2b plus ribavirin for initial treatment of chronic hepatitis C: A randomised trial. Lancet 358: 958-965, 2001.

8. Bussel JB and Marks KM: How effective is eltrombopag for the treatment of thrombocytopenia in patients with $\mathrm{HCV}$ infection? Nat Clin Pract Gastroenterol Hepatol 5: 424-425, 2008.

9. Cholongitas E, Papatheodoridis GV, Vangeli M, Terreni N Patch D and Burroughs AK: Systematic review: The model for end-stage liver disease - should it replace Child-Pugh's classification for assessing prognosis in cirrhosis? Aliment Pharmacol Ther 22: 1079-1089, 2005.

10. Lackner C, Struber G, Bankuti C, Bauer B and Stauber RE: Noninvasive diagnosis of cirrhosis in chronic hepatitis $\mathrm{C}$ based on standard laboratory tests. Hepatology 43: 378-379, 2006.

11. McHutchison JG, Dusheiko G, Shiffman ML, Rodriguez-Torres M, Sigal S, Bourliere M, Berg T, Gordon SC, Campbell FM, Theodore D, et al; TPL102357 Study Group: Eltrombopag for thrombocytopenia in patients with cirrhosis associated with hepatitis C. N Engl J Med 357: 2227-2236, 2007.

12. O'Brien PC and Fleming TR: A multiple testing procedure for clinical trials. Biometrics 35: 549-556, 1979.

13. Manns M, Wedemeyer $\mathrm{H}$ and Cornberg M: Treating viral hepatitis C: Efficacy, side effects and complications. Gut 55: 1350-1359, 2006.

14. Gutfreund $\mathrm{K}$ and Bain V: Chronic viral hepatitis C: Management update. CMAJ 162: 827-833, 2000.

15. Ikezawa K, Naito M, Yumiba T, Iwahashi K, Onishi Y, Kita H, Nishio A, Kanno T, Matsuura T, Ono A, et al: Splenectomy and antiviral treatment for thrombocytopenic patients with chronic hepatitis C virus infection. J Viral Hepat 17: 488-492, 2010.

16. Neunert C, Lim W, Crowther M, Cohen A, Solberg L Jr and Crowther MA; American Society of Hematology: The American society of hematology 2011 evidence-based practice guideline for immune thrombocytopenia. Blood 117: 4190-4207, 2011

17. Provan D, Stasi R, Newland AC, Blanchette VS, Bolton-Maggs $P$, Bussel JB, Chong BH, Cines DB, Gernsheimer TB, Godeau B, et al: International consensus report on the investigation and management of primary immune thrombocytopenia. Blood 115: 168-186, 2010

18. Bussel JB, Buchanan GR, Nugent DJ, Gnarra DJ, Bomgaars LR, Blanchette VS, Wang YM, Nie K and Jun S: A randomized, double-blind study of romiplostim to determine its safety and efficacy in children with immune thrombocytopenia. Blood 118: 28-36, 2011.

19. Tiede MP, Ahn ER, Jy W, Scagnelli T, Bidot CJ, Horstman LL, Jimenez JJ and Ahn YS: Life-threatening hypercoagulable state following splenectomy in ITP: Successful management with aggressive antithrombotic therapy and danazol. Clin Appl Thromb Hemost 11: 347-352, 2005.

20. Crary S and Buchanan G: Vascular complications after splenectomy for hematologic disorders. Blood 114: 2861-2868, 2009.

21. Blanchette V and Bolton-Maggs P: Childhood immune thrombocytopenic purpura: Diagnosis and management. Hematol Oncol Clin North Am 24: 249-273, 2010.

22. Deutsch VR and Tomer A: Megakaryocyte development and platelet production. Br J Haemotol 134: 453-466, 2006.

23. Temel T, Cansu DU, Temel HE and Ozakyol AH: Serum thrombopoietin levels and its relationship with thrombocytopenia in patients with cirrhosis. Hepat Mon 14: e18556, 2014.

24. Wang ES, Lyons RM, Larson RA, Gandhi S, Liu D, Matei C, Scott B, Hu K and Yang AS: A randomized, double-blind, placebo-controlled phase 2 study evaluating the efficacy and safety of romiplostim treatment of patients with low or intermediate-1 risk myelodysplastic syndrome receiving lenalidomide. J Hematol Oncol 5: 71, 2012. 